Evans, D. G. \& Wardlaw, A. C. (1953). J. gen. Microbiol. 8, 481-487.

\title{
Gelatinase and Collagenase Production by certain Species of Bacillus
}

\author{
By D. G. EVANS and A. C. WARDLAW \\ Department of Bacteriology, University of Manchester
}

\begin{abstract}
SUMMARY : Fifty-three strains from six species of the genus Bacillus were tested for their ability to produce gelatinase and to disintegrate the collagen matrix of bone. Some strains of $B$. cereus and $B$. anthracis produced a true collagenase which brought about complete disintegration of the collagen substrate, while strains of $B$. subtilis, $B$. licheniformis, $B$. brevis and $B$. megaterium were either completely inactive or produced only partial disintegration. Collagenase production was always accompanied by gelatinase production but the reverse was not the case. Concentrated culture filtrates of $B$. cereus were shown to contain collagenase and gelatinase, whereas those of $\boldsymbol{B}$. subtilis contained gelatinase only.
\end{abstract}

In a previous communication (Evans \& Wardlaw, 1952)' we described some of the properties of proteolytic culture filtrates from a strain of Bacillus subtilis. The culture filtrates were highly active in liquefying gelatin and in disintegrating hide powder which is a denatured form of skin collagen. The filtrates were, however, unable to break down undenatured tendon collagen either in the form of thin slices or thin sheets prepared from soluble collagen by the method of Delaunay, Guillaumie \& Delaunay (1949). Bacillus strains have, however, been shown to attack the collagen-like matrix of tooth dentine (Evans \& Prophet, 1950), and this has led us to extend our previous investigation. We have now tested the ability of a number of bacillus strains to produce collagenase, using as substrate a finely divided preparation of decalcified bone. This preparation was not regarded as a pure substrate but was nevertheless a rich source of native collagen. The strains were also examined for their ability to produce gelatinase, and a study was made of the relationship between this enzyme and collagenase.

\section{MATERIALS AND METHODS}

Preparation of collagen substrate. Limb-bone shafts from adult rabbits were freed from marrow and connective tissue and dried over $\mathrm{P}_{2} \mathrm{O}_{5}$. After preliminary fragmentation the bone was ground to a fine powder in a low-temperature ball mill as used by Evans \& Prophet (1950) for the preparation of powdered dentine. The powder was decalcified by suspension in a large volume of $0 \cdot 2 \mathrm{~N}-\mathrm{HCl}$ for $1 \mathrm{hr}$. and then centrifuged. The deposit was washed free from acid by resuspending in water and centrifuging several times; the final suspension was dialysed against running water and dried from the frozen state to yield a very finely divided preparation of decalcified bone.

Test for collagenase production. The decalcified bone powder was sterilized by exposing thin layers to ultra-violet radiation. A saline suspension of the powder was mixed with melted $3 \%$ agar in saline which had been cooled 
below $45^{\circ}$ and the mixture poured into Petri dishes. For each plate $10 \mathrm{ml}$. of agar and $20 \mathrm{mg}$. of decalcified bone powder were used. Disks $6 \mathrm{~mm}$. in diameter were cut from the agar plate with a sterile cork-borer and transferred to tubes containing $10 \mathrm{ml}$. sterile $1 \%$ Evans peptone in saline at $\mathrm{pH} \mathbf{7 \cdot 6}$. Two disks were placed in each tube. The tubes of medium after the addition of the disks were incubated for 1 week to check sterility; they were then inoculated with the bacillus strains listed in Table 1 and incubated at $37^{\circ}$. With aseptic precautions one disk was removed from each culture after 3 days, the other disk after 5 days and each examined microscopically at $\times 15$ magnification. The degree of disintegration of the collagen particles was determined by comparison with a control disk from a tube of uninoculated medium and was estimated as none, partial or complete (P1. 1). No attempt was made to describe grades of partial clearing.

With some of the strains the collagenase activity of culture filtrates was titrated. Serial dilutions of the filtrates were made in normal saline, and to each was added one collagen-agar disk. The tubes were incubated at $37^{\circ}$ for $18 \mathrm{hr}$. and the end-point taken as the highest dilution which gave complete clearing of the disk. The reciprocal of the end-point dilution gave the number of minimal collagenase doses/ml. (m.c.d./ml.).

Test for gelatinase production. The same cultures which were being tested for collagenase activity and which contained collagen-agar disks, were also tested for their gelatinase activity. After 2 and 5 days of incubation, $1 \mathrm{ml}$. samples of culture were removed and the gelatinase activity titrated by the settingtime method (Evans \& Wardlaw, 1952). Serial dilutions of culture were made in saline, and to each was added an equal volume of $5 \%$ gelatin solution. This concentration of gelatin we found more sensitive than the $10 \%$ solution used previously. The mixtures were incubated for $1 \mathrm{hr}$. at $37^{\circ}$ and then cooled in ice water. The time taken for each mixture to set was determined and the end-point taken as that dilution which gave the same setting time as a control solution of $1.5 \%$ gelatin alone. The activity of a culture was expressed as the number of minimal gelatinase doses/ml. (m.g.d./ml.) which was the reciprocal of the end-point dilution. Culture filtrates were titrated in a similar manner.

Strains. The fifty-three strains used are given in Table 1. They were obtained as named species from the National Collection of Type Cultures (NCTC), from the Culture Collection of the Wellcome Research Laboratories (CN) and from our own departmental collection (DBM). In addition, Dr G. P. Gladstone supplied us with three strains of $B$. anthracis-M36, Sterne and NPA. We accepted the identity of the strains as received and from the first cultures prepared fresh batches of freeze dried cultures.

\section{RESULTS}

\section{Gelatinase and collagenase in cultures}

The results of the tests on the fifty-three different strains are shown in Table 1. Species were found to differ considerably in their ability to produce gelatinase. $\boldsymbol{B}$. anthracis and $\boldsymbol{B}$. subtilis were the most active, some strains of which gave titres of 100-300 m.g.d./ml. after 5 days, whereas no strains of $B$. brevis and 
Table 1. The ability of fifty-three Bacillus strains from six different species to produce gelatinase and collagenase

Collagenase activity

Gelatinase activity Degree of disintegration

Bacillus species and strain no.

$\underbrace{\text { (m.g.d./ml.) }}_{\text {days }} \underbrace{}_{5 \text { days }}$

$\overbrace{3 \text { days }}^{\text {of collagen }} \underbrace{}_{5 \text { days }}$

B. subtilis:

$\left\{\begin{array}{l}\text { NCTC } 3610 \\ \text { NCTC } 6276 \\ \text { NCTC 6346 } \\ \text { NCTC 7241 } \\ \text { CN 788 } \\ \text { CN 789 } \\ \text { CN } 808 \\ \text { CN } 831 \\ \text { CN } 1508 \\ \text { CN } 1576\end{array}\right.$

$120 \quad 90$

$30 \quad 60$

24

$50 \quad 50$

$30 \quad 25$

$12 \quad 18$

$\begin{array}{rr}12 & 18 \\ 4 & 10\end{array}$

140

25

25

NCTC 962

B. licheniformis :

NCTC 1024
NCTC 1025

$\{$ NCTC 1026

NCTC 1027

NCTC 1097

NCTC 6349

CN 753

CN 2194

B. cereus:

$\left\{\begin{array}{l}\text { CN 2484 } \\ \text { CN 2501 }\end{array}\right.$

CN 2503

CN 2504

NCTC 926

NCTC 6093

CN 1409

B. cereus var. mycoides:

CN 1541

CN 2195

CN 2495

CN 2500

CN 2521

NCTC 1712

NCTC 2620

DBM 121

B. anthracis:

DBM 122

DBM 123

M36

Sterne

NPA

NCTC 7577

CN 2904

B. brevis :

CN 2918

CN 2922

CN 2934

CN 3005

NCTC 2605

NCTC 5636

B. megaterium :

$$
\text { CN } 737
$$

CN 1759

CN 2496

CN 2529

A titre of $<1$ means that the undiluted culture showed no activity. Disintegration of collagen: $0=$ none; $+=$ partial $;++=$ complete.

NCTC $=$ National Collection of Type Cultures, Colindale, London, N.W. 9.

$\mathrm{CN}=$ Wellcome Research Laboratories, Beckenham, Kent.

DBM = Department of Bacteriology, Manchester. 
B. megaterium showed detectable activity. The strains within a single species also showed marked differences in gelatinase production. With B. subtilis strains, for example, the titres ranged from 4 to 130 , and with $B$. anthracis there were even greater differences, from 3 to 300 . With many strains the gelatinase activity at 2 and 5 days was similar, although in some cases, as with $\boldsymbol{B}$. anthracis and $\boldsymbol{B}$. cereus, the 5-day cultures showed a considerably higher titre. Although it was not possible with some of the strains to detect gelatinase by the titration method, this did not imply that the strains were entirely devoid of the ability to produce the enzyme. It was possible to detect gelatinase action by these strains in gelatin stab cultures incubated for long periods.

The collagenase tests showed that some strains produced complete disintegration of the collagen particles in the disks while other strains were completely inactive. The greatest activity was shown by B. cereus; of the seven strains tested, complete disintegration was shown by five of them in 3-day cultures and by six in 5-day cultures. The $\boldsymbol{B}$. anthracis strains were also able to produce complete disintegration, although they were not so rapidly active as those of $B$. cereus. None of the eight strains of $B$. anthracis showed activity in 3-day cultures, but after 5 days of incubation four of them had produced complete disintegration and three partial disintegration. Many of the strains of other species showed partial effects, but complete disintegration was given only by two strains of $B$. cereus var. mycoides. No detectable collagenase activity was shown by any of the strains of $B$. brevis or B. megaterium.

It was evident that in the group as a whole there was some correlation between collagenase and gelatinase production. Those strains which produced collagenase always produced gelatinase, and those which showed no gelatinase activity were also inactive towards collagen. The correlation was, however, by no means complete, for gelatinase production was not always accompanied by collagenase production. Further, with those strains which produced both enzymes, there was no quantitative correlation between gelatinase activity and rapidity with which collagen was disintegrated. This lack of association between the two enzyme activities was most apparent with strains of B. subtilis and B. cereus. For example, strain NCTC 7241 of B. subtilis had a gelatinase titre of 50 but showed no disintegration of collagen at 3 days. On the other hand, strain NCTC 6349 of $B$. cereus produced complete disintegration of collagen at 3 days, although the gelatinase titre was only 8 . This strongly suggested that the proteolytic enzymes in the two cultures differed considerably in their substrate specificity, and in order to obtain more conclusive evidence of these differences further tests were made with concentrated culture filtrates.

\section{Gelatinase and collagenase in filtrates}

Concentrated culture filtrates were prepared from two strains of each of B. subtilis and $B$. cereus by the method we previously used (Evans \& Wardlaw, 1952). Cultures were filtered after 3-4 days growth and the filtrates precipitated by adding $500 \mathrm{~g}$. ammonium sulphate/l. The precipitate was dialysed 
to remove ammonium sulphate and then dried from the frozen state. To be tested for enzyme activity the dried preparations were dissolved in saline to give approximately $1 \%(\mathrm{w} / \mathrm{v})$ solutions. Each preparation was titrated for gelatinase and collagenase, and the results are given in Table 2. To facilitate comparison of the two activities, the gelatinase titres of $B$. cereus have been adjusted to equal those of $\boldsymbol{B}$. subtilis and the collagenase titres scaled accordingly. Neither of the $B$. subtilis filtrates, although highly active in liquefying gelatin, were able to bring about complete clearing of the collagenagar disks. The two $B$. cereus filtrates, on the other hand, with the same gelatinase activity as those of $\boldsymbol{B}$. subtilis, were able completely to clear the disks even at dilutions of $1 / 260$ and $1 / 64$. These results are in agreement with those obtained in the tests with cultures and clearly show that the proteolytic enzymes of these two species differ in their substrate specificity.

Table 2. Comparison of the gelatinase and collagenase activities of culture filtrates of Bacillus subtilis and $\mathrm{B}$. cereus

$\begin{array}{ccc}\text { Filtrate } & \begin{array}{c}\text { Gelatinase titre } \\ \text { (m.g.d./ml.) }\end{array} & \begin{array}{c}\text { Collagenase titre } \\ \text { (m.c.d./ml.) }\end{array} \\ \text { B. subtilis: NCTC 3610 } & 300 & <1 \\ \text { CN 831 } & 300 & <1 \\ \text { B. cereus }: \text { NCTC 6349 } & 300 & 260 \\ \text { CN 2504 } & 300 & 64\end{array}$

The results also suggest that with $B$. cereus two enzymes are involved in attacking gelatin and collagen. The two $B$. cereus filtrates attacked gelatin to the same titre but there was a four-fold difference in their collagenase titres. If the enzyme which attacked gelatin was also responsible for the breakdown of collagen, then it would have been expected that the collagenase titres of the two filtrates would have been the same. The fact that they were considerably different strongly suggests that two independent enzymes were present in the filtrates, one which attacked collagen and possibly gelatin, and one which attacked gelatin only.

\section{The nature of the collagen substrate}

It is well known that collagen can undergo changes in its susceptibility to enzyme action. We found that heating a watery suspension of decalcified powdered bone at $65^{\circ}$ for $10 \mathrm{~min}$. considerably increased its susceptibility, so that it was completely disintegrated by enzyme preparations of $\boldsymbol{B}$. subtilis which were unable to attack the unheated material. On the other hand, the bone powder was made completely resistant to enzyme action by treating it with low concentrations of formaldehyde or tannic acid. Not only did this treatment make the collagen resistant to bacillus collagenases but also to the more rapidly acting collagenase of Clostridium histolyticum. In view of this, care was taken in preparing the bone substrate to maintain the collagen in its unaltered native form. It was not possible to use untreated powdered bone as the collagen substrate, for bone in its calcified form was found to be completely unaffected by bacillus and clostridium collagenases. A similar observation 
was made by Evans \& Prophet (1950) with the collagen-like matrix of dentine. It was therefore necessary to decalcify the bone and $0 \cdot 2 \mathrm{~N}-\mathrm{HCl}$ was used for this purpose. This treatment, apart from removing the calcium salts and thereby exposing the collagen matrix to enzyme attack, probably had little or no denaturing effect on the collagen, for it was found that bone powder decalcified with phosphate buffer at $\mathrm{pH} \mathrm{6.0,} \mathrm{a} \mathrm{mild} \mathrm{treatment} \mathrm{hardly} \mathrm{likely}$ to denature the collagen, was just as sensitive to enzyme attack as bone decalcified by $0 \cdot 2 \mathrm{~N}-\mathrm{HCl}$.

An experiment was also made which showed that the method we used for sterilizing decalcified bone powder by ultra-violet radiation did not alter its susceptibility to enzyme. Titrations of collagenase preparations made with agar disks containing irradiated decalcified bone powder gave the same endpoints as titrations made with disks containing powder which had not been exposed to radiation.

As a result of these tests we considered that the substrate we used was native collagen which had not been denatured during its preparation. If this be accepted, then those bacillus strains which were able to disintegrate this substrate were true collagenase producers.

\section{DISCUSSION}

Although many bacteria are known to attack denatured collagen in the form of gelatin, relatively few species have been shown to break down native collagen. Our experiments indicate that some strains in the genus Bacillus are able to produce a true collagenase. It is possible that we have been able to detect this enzyme because of the finely divided form of substrate we have employed. The substrates commonly used in testing collagenase action are thin slices of tendon or thin sheets of precipitated collagen ('collagen paper'). These substrates are comparatively insensitive indicators and are not disintegrated by such high dilutions of enzyme as is collagen powder prepared from bone. With our most potent concentrated filtrates from $B$. cereus, we showed that agar disks containing collagen powder as indicator were at least 30 times more sensitive than collagen paper. Similar results were obtained with the collagenase of $\mathrm{Cl}$. histolyticum. Decalcified bone we have found can be prepared as a very fine powder, and thus when treated with enzyme is intimately exposed to its action. It is possible that the collagen-agar disk method may be of value in detecting unsuspected collagenase production by other bacterial species.

It is not yet known whether the bacillus collagenases are of the same kind as those produced by $\mathrm{Cl}$. welchii type A (Oakley, Warrack \& van Heyningen, 1946) and $C l$. histolyticum (Oakley \& Warrack, 1950). The clostridium enzymes are able to break down with comparative ease the more resistant collagenase indicators such as collagen paper and even pieces of skin. This is due to the fact that they are produced in high concentration in culture and does not necessarily imply that they are of a different kind from the collagenases of the bacillus group. The collagenases of the two groups do, however, appear to differ in the rate at which they act on the substrate. We have shown, with 

Journal of General Microbiology, Vol. 8, No. 3
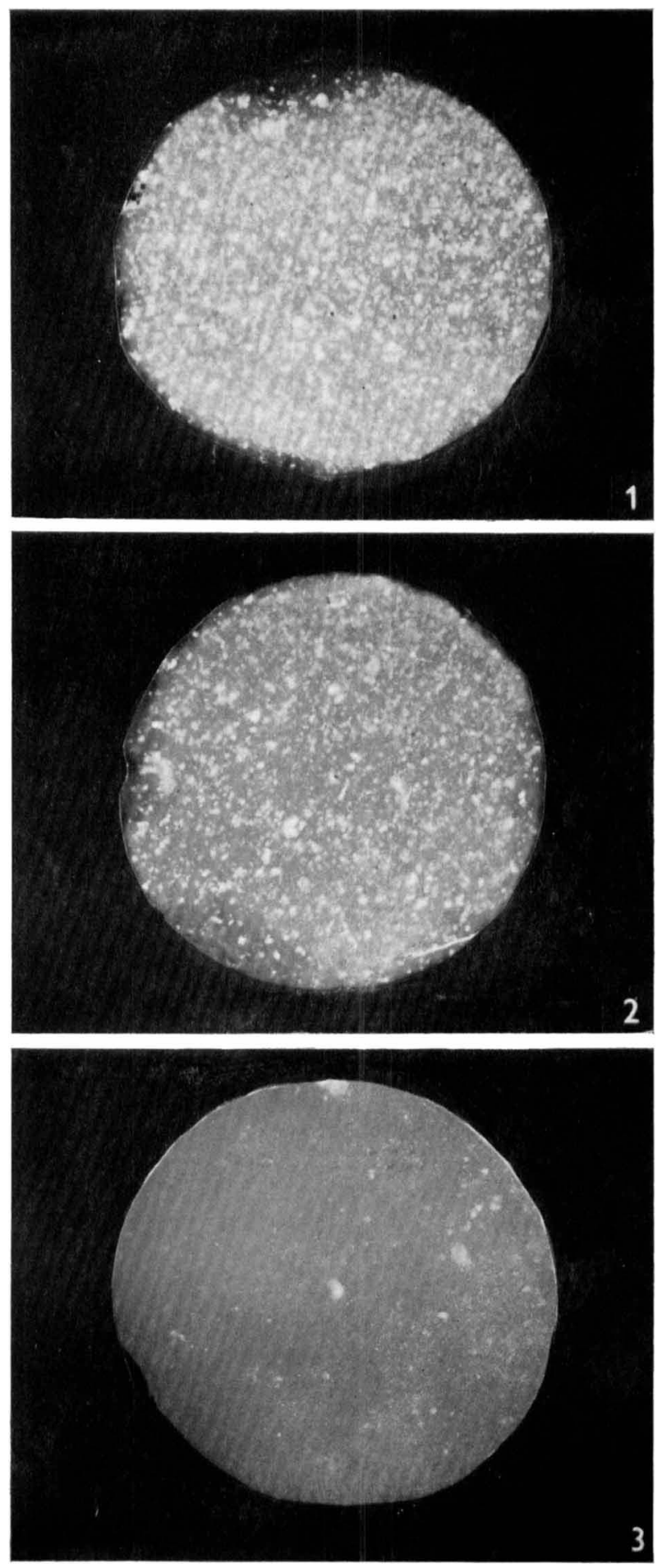

D. G. Evans \& A. C. Wardiaw-Gelatinase and collaglenase of Bachllous Spp. Plate 1 
collagenase preparations from each group, containing the same number of m.c.d./ml., that the disintegration of bone powder is completed by the clostridium collagenases in less than half the time taken by those of the bacillus group.

The experiments have also shown that the collagenase activity of bacillus strains is not dependent on their gelatinase activity. Some strains with low gelatinase activity are highly active in disintegrating collagen, whereas others with high gelatinase activity have little or no effect on collagen. Even in the case of those culture filtrates of $B$. cereus which actively attack both gelatin and collagen, the results indicated that two independent enzymes are involved, one which attacks collagen and possibly gelatin, and one which attacks gelatin only. In this respect there is an analogy with the collagenase ( $\kappa$-antigen) and gelatinase ( $\lambda$-antigen) of $\mathrm{Cl}$. welchii (Oakley, Warrack \& Warren, 1948). The analogy, however, is by no means complete, for we have been unable so far to obtain conclusive evidence that either the gelatinase or the collagenase of $B$. cereus is antigenic. Further attempts are being made to obtain antibodies to these enzymes, for we consider that if it be possible to use serological tests, the proteolytic enzymes of this group will then be more clearly differentiated.

We should like to express our thanks to Mr H. Proom and Dr G. P. Gladstone for strains and to Dr G. H. Warrack for rat collagen paper. One of us (A.C.W.) wishes to acknowledge the receipt of a Scholarship from the Medical Research Council.

\section{REFERENCES}

Delaunay, M., Guillaumie, M. \& Delaunay, A. (1949). Études sur le collagène. 1. A propos des collagènases bactériennes. Ann. Inst. Pasteur, 76, 16.

Evans, D. G. \& Prophet, A. S. (1950). Disintegration of human dentine by bacterial enzymes. Lancet, i, 290.

Evans, D. G. \& Wardlaw, A. C. (1952). In vitro and in vivo properties of culture filtrates of Bacillus subtilis with high gelatinase activity. J. gen. Microbiol. 7, 397.

OAKLEY, C. L. \& WARraCK, G. H. (1950). The alpha, beta and gamma antigens of Clostridium histolyticum (Weinberg \& Séguin, 1916). J. gen. Microbiol. 4, 365.

Oakley, C. L., Warrack, G. H. \& Heyningen, W. E. van (1946). The collagenase ( $\kappa$-toxin) of Cl. welchii type A. J. Path. Bact. 58, 229.

OAKLey, C. L., WArrack, G. H. \& Warrex, M. E. (1948). The kappa and lambda antigens of Clostridium welchii. J. Path. Bact. 60, 495.

\section{EXPLANATION OF PLATE}

Collagen powder, suspended in agar disks, showing different degrees of disintegration after exposure to bacillus cultures. (1) no disintegration, (2) partial, (3) complete. $\times 9$.

(Received 17 December 1952) 